\title{
A Web-Based System to Facilitate Elders Communication with Their Families Living Abroad
}

\author{
Pedro C. Santana ${ }^{1}$, Marcela D. Rodríguez ${ }^{2,1}$, Víctor M. González ${ }^{4}$, Luis A. Castro ${ }^{2}$, Ángel G. \\ Andrade $^{3,1}$, Jesús Favela ${ }^{2}$ \\ ${ }^{1}$ School of Engineering, UABC University, México \\ ${ }^{2}$ Computer Science Depart., ${ }^{3}$ Elec. and Telecomm. Depart., CICESE Research Center, México \\ ${ }^{4}$ Department of Informatics, University of California, Irvine, USA \\ \{psantana, marcerod, quiroa, aandrade,favela\}@cicese.mx,vmgyg@ics.uci.edu
}

\begin{abstract}
The aging of the population is a phenomenon faced by most nations. Growing old is often accompanied of a loss of close companionship which has been shown may aggravate the cognitive impairment of elders. From a qualitative study, key issues emerged regarding unmet needs of elders communication that we propose to address with web-based technology. We decided to create an electronic family newspaper to incorporate elders to the current social networks created by their younger relatives who already communicate through Internet applications, such as $I M$ and e-mail. The system uses web-based technology to make it accessible from any web browser for those users living abroad. To serve the needs of elders and make the input of information easier, several autonomous agents help the user to interact with the system that can be accessed by any electronic display with a touch screen, such as a Tablet PC.
\end{abstract}

\section{Introduction}

The aging of the population is a phenomenon faced by many nations, such as Mexico, in which $7.5 \%$ of the population is 60 years or older. It is estimated that by 2030 this figure will be more than double, reaching $17.5 \%$ [1]. Among those elders, $10 \%$ of them live alone with no close family members around them. This condition is more likely to occur in some regions of Mexico as it is related to the ever increasing migration of one or more of their relatives to the USA. The living conditions of those elders can be quite complex as they often face the impossibility of visiting or being visited by their families as they lack proper documentation (VISAS or residency permits). And even when this is not a problem, distance and cost might reduce direct contact to one visit every other year. As it has been found, lack of contact with family members and friends may have a negative impact on elder's health, such as accelerating a cognitive decline $[2,3]$. Thus, Mexican elders in this situation face particular challenges that might aggravate some of the wellknown effects of living with no close companionship.

Technically, the system focuses on solving two important challenges. On the one hand, the system aims to provide adequate interfaces for each of the different types of users that would use it. Elders must have an interface that is adequate for their needs. Family members must have means to use their current tools (e.g. email clients) without the need to migrate to another communication tool. On the other hand, the system enhances the interactions with family members. Based on a set of autonomous agents the system provides an enhanced level of interaction beyond synchronous and asynchronous forms.

Our work aims to provide a technological solution focused on supporting the relationship between elder people living alone in Mexico and their relatives living abroad. The central concept of the proposed system is an electronic family newspaper, through which elders and their families: a) share important information, b) personal reminiscences and cultural stories c) may interact with virtual relatives; and c) occasionally may interact with the real relatives. It is thought that the electronic family newspaper will enable elders to feel more engaged and connected with their relatives living abroad as it provides richer forms of communication, and, it will facilitate their integration into the networks that currently connect members of their families who increasingly are making use of e-mail systems to keep in touch with each other and help to incorporate elders to the current social networks created by their younger 
relatives who already communicate through Internet applications, such as IM and e-mail.

\section{Problem context: the need to be connected}

An initial understanding of the phenomenon of migration and the need to be connected was obtained by the analysis of the records of the websites of Alhuey (see Figure 1), San Luis de la Paz and Cihuatlán, all small towns in different parts of Mexico with a important number of people living abroad or in other big cities in Mexico. Members of these communities maintain contact with their roots through these Internet applications which enables them to share photos, stories and anecdotes. We analyzed the contents of those web sites as a departure point to understand some of the aspects involved in migrating and keeping ties to communities.

A central feature of these web sites is that they let people of different ages share personal information in the form of digital photos. For instance, in Alhuey's web site visitors can upload pictures and post comments to share with others. We found that in this site an average of 2 pictures and 6 posts are placed every single day by around 1050 users.

Based on the comments posted on the web sites, we found that some of them have been absent from their native land for years. Most of them are interested in what is happening in their towns and some of them want to know what happened to their old friends. For instance, they are concerned about issues such as people getting married, people who passed away, whether some of their friends have children or not, but most of them just want to know where everybody is living. Consequently, the web site plays a major role on keeping people in touch with their social networks from their beloved town.

We also noticed that several users are using these websites as communication tools to complement other tools such as e-mail or instant messaging. The sense of community facilitated by the technology, perhaps particular for small towns where everyone knows each other, provided good initial insight of what it is required by people in order to be connected in spite of distance. These results guide our initial understandings that serve to focus our inquiry over the particular situation of elders living alone.

\section{Understanding elderly people}

Our research was then oriented towards understanding the emotional needs of Mexican elders with families living abroad. We wished to gain knowledge about the experiences of elders in regards to the following five main aspects: communication with relatives, feelings of isolation, health care, keeping up to date with things around them such as family events, and being independent. From these lines of inquiry we aimed to reveal fundamental requirements for a system supporting elders.

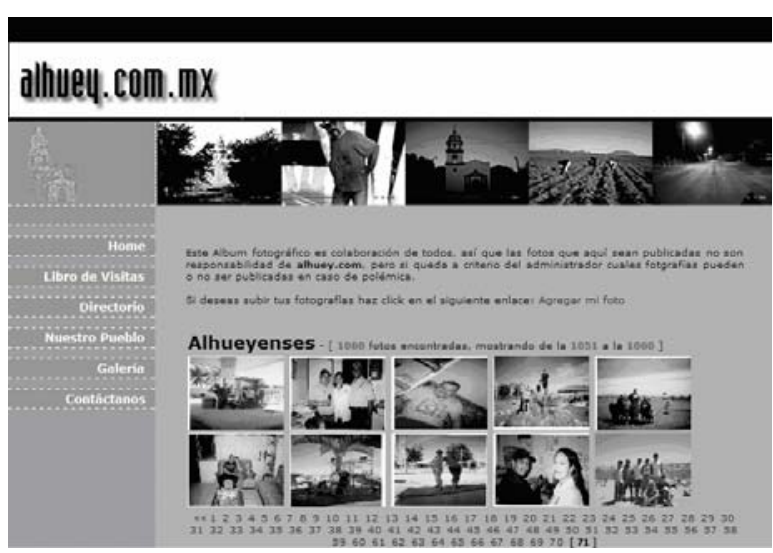

Figure 1. Alhuey web site Photographs in Album.

\subsection{Methods}

Based on personal contacts and recommendations from them, we contacted a number of elders that were experiencing the scenario that we were aiming to understand. Our set of informants included people of different genders, age and living in different geographical regions of Mexico. Our interviews were semi structured and were conducted within the home environment (e.g. the kitchen) following standards and recommendations for qualitative interviews [4].

\subsection{Results}

The interviews were analyzed using a comparative verification of evidence which resulted in the identification of major themes for each topic of inquiry. In this section we explained some of the most relevant results.

We found that the main mode of communication with relatives living abroad was the telephone which basically is used to update each other about news and recent family events. Phone calls are not always frequent and are more likely to occur at special occasions such as birthdays or holidays or when some emergency issues arise (e.g. accidents or other major problems). All our informants expressed their preference for being in constant communication with their families, but recognized that this is not always possible. One of our interviewees expressed that the impossibility of communicating on a more regular basis can bring some sadness to her. Those facts point to the relevance of providing appropriate mechanisms 
to help elders feel connected to their families. We noticed that a central component that is missed whenever families get separated is the ability to share day-to-day experiences. Many times the content of communications is limited to basic information regarding the well-being of the persons, health, financial situation, or relevant events. However elders and their families have little chance to share the little things that sometimes make life enjoyable and they used to share when they were together: local events, conversations, the ability to see each other and other emotionally important facts.

Our results also highlighted the importance given by elderly people to photo albums. It is a real treasure to them. An old couple commented, "Our grandchildren love to look at the album and asks us what her mother used to do when she was a kid". Moreover, they intend to acquire a video camera to record all the visits of their relatives. This showed us that pictures are artifacts from which we could take advantage of, due to the stories and emotive load associated to them.

Finally, it was interesting to find that those elders interviewed show disposition to engage in learning new things. As a way to keep them active, some of them are taking courses (e.g. English lessons). Similarly, another person is going to elementary school and is very proud of her achievements and motivated to continue her studies for as long as she can. This disposition to learn can be very relevant for the purposes of introducing any technological solution.

Based on these findings and our preliminary understanding of the phenomenon, we engaged in designing a system to support the emotional ties among Mexican elders and their families living abroad, focusing on a way to enhance their communication.

\section{Related work}

In this section we compare some alternative approaches that have been discussed within the context of providing companionship for elders. Some design concepts and products have intended to create emotional connections over a distance by applying theories of affective computing combined with ubiquitous computing technology. For instance, the Gust of Presence system provides a suitable carrier for affective communication by enabling a two-way notification of presence [5]. This system lets parents and children who live apart inform each other when they have arrived home. It uses a bowl, which senses when the user throws something into it, such as money or keys, which may indicate he has arrived at his home. Then, the bowl takes a picture from the inside and sends this information to another identical bowl located in the parent's home. Similarly, the Lovelet [6] is a wearable communication tool for intimate people for naturally and timely conveying affection. This consists of a thermo sensor that always senses air temperature surrounding a user, the temperature data is transmitted to another user and depending on the temperature, a full color LED (Light Emitting Diode) illuminates in different color to indicate an emotional state. Although, both the Gust of Presence and the Lovelet can help to share emotional states, they are not appropriate to support communication in a more direct way and do not address directly the needs of elders.

The SeniorNet [7] tool is a computer-mediated communication (CMC) tool exclusively for older adults and was used to identify a number of motivations that older adults expressed for using CMC technology. Many SeniorNet users mentioned that SeniorNet was an important way to meet people with similar interest, access information, and develop companionship and supportive relationships. Although SeniorNet seems to be in the right track attending the needs of elders, it fails to be oriented towards supporting the communication with people outside the network that include family members using other tools such as e-mail.

In contrast with those systems, we aimed to create a system that provides a more comprehensive support for elders and their families. Based on our interviews we decided to focus the design of our systems to ameliorate the isolation of elders by means of digital photos and narrations accessible through a repository of information in the form of an electronic newspaper. We decided to use the metaphor of a newspaper as it is a well understood concept by both elders and young people and conveys the idea that information is provided in a frequent basis. We envisioned that through the newspaper, the elders and their family would share their emotions, anecdotes, memories, and other kind of information, such as Mexican traditions.

\section{Envisioned system}

We envision that to ameliorate the loneliness of Mexican elders, our system needs to address the following aspects:

Enable elders and their family to feel close and maintain contact in an entertaining way. We envisioned an electronic family newspaper, which enables users to share information, such as personal memories, anecdotes, or traditions that elders would like to transmit to their younger relatives, or vice versa, the family residing in USA sharing the American customs they have adopted. The information 
transmitted through the family newspaper, is categorized in different sections. For instance, in the Social section the family may publish photos or a video of when the granddaughter graduated from high school. The Entertainment section provides activities for elders, such as a memory game, in which the elder may play with a virtual relative.

Enable an easy way for elders to use the system. We consider that for elders we need to propose an easy way to use the system based on a computer with a touch screen. The system enables elders to contribute to build the newspaper by using digital cameras, and scanners to get images of documents or printed photos they want to publish in the newspaper.

Make the system accessible for the elders' family. As most of the elders' families already use Internet communication tools to be in contact with their relatives and friends, we should take advantage of this. Thus, with the aim of providing an easy to use system, it integrates web technology already known by the users living abroad.

Elders may access and use the system in several places within the home. The family newspaper is presented in a display that can be hung on any wall of any room of the home of an elder, in which a senior spends most of his time or usually reads the conventional newspaper.

To clarify how these features are addressed by our system, we constructed several scenarios of use to illustrate the system's functionality. The creation of scenarios enabled us to generate and communicate design ideas for our system and to better understand the implications of particular design solutions [8]. Next, we present some of the use scenarios in which we envision how elders may ameliorate their loneliness through the electronic family newspaper.

\subsection{Scenario 1}

Mrs. and Mr. Valenzuela are old adults living alone in Guadalajara, Mexico with two daughters and a son living in Santa Ana, California, USA. While Mrs. Valenzuela is preparing breakfast, the display in the kitchen plays an alarm to notify her that there is some family news that may be interesting for her. She approaches the display and notices that there are new messages in the Cooking section. As the "5 de Mayo" Mexican holiday is coming, her daughters are organizing a dinner at the neighborhood and have published a list of potential Mexican dishes (see Figure 2) they would like to prepare for the occasion. Mrs. Valenzuela realizes that she has the recipes of some of them and decides to go for her cooking book to send them to her daughters. While Mrs. Valenzuela goes for the book, Mr. Valenzuela quickly pulls the display and reads the family newspaper while he is eating breakfast. He selects the Sports section because he is sure his son Mario has written a review of the latest soccer game of the Mexican league. As Mr. Valenzuela realizes that his son would like to read interviews of some of the players that were published in yesterday's local newspaper, he scans the note and attaches it to the review. At that moment Mrs. Valenzuela is back with a bunch of old cooking books and asks her husband: "Do you think that they will find Tejocotes for the tea in Santa Ana?."

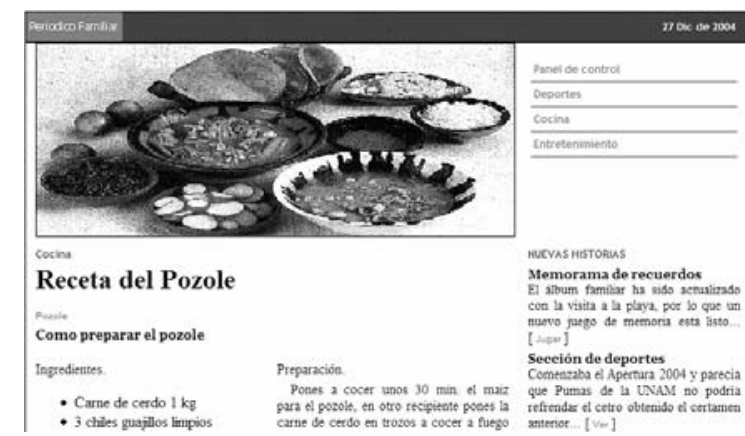

Figure 2. Family Newspaper - Cooking section.

\subsection{Scenario 2}

Mrs. Diana is a 72 years old woman who lives alone in Tijuana, Mexico. She likes to play with the memory game included in the Entertainment section of her family newspaper. When she selects the memory game to start to play, a set of images of her family residing in the USA and other places of Mexico is presented at the top of the screen. As she misses her grandson Jose, she decides to play this session with him. For this, she selects the photograph of Jose and the virtual Jose appears saying hello and the memory game, which includes only images of the latest events related to her grandson. When Diana matches a first pair of cards, the virtual Jose, explains a little bit of the event in the picture. While Mrs. Diana and the virtual Jose are playing, her grandson is doing his homework on his computer. He realizes that his grandmother is playing and decides to join the game. The grandmother is glad of playing with her grandson.

\section{System design}

In order to achieve the system functionality we are proposing an agent-based system which architecture is described next.

\subsection{Dual interfaces}

The system was designed to provide an interface for elders and another interface for family members. To serve the needs of elders and make the input of 
information easier, the system is based on Tablet PC technology. By using a pen to touch the screen, elders can access the functionality and input information. In addition, Family members can use the system through any other device with a web browser. We believe that this would help the seamless adoption of the system.

\subsection{System Architecture}

As illustrated in Figure 3, the architecture of the electronic family newspaper consists of several layers.

Codice CMS. The system includes a Weblog Content Management System named Codice in which a weblog is created by the family members to load the information they want to publish in the family newspaper through the Codice Web Services APIs. A weblog is a term used to refer to a webpage that has frequent postings made to it by the person who created the page and others who are given rights to access the page. The trend of using weblogs is gaining momentum with the introduction of automated publishing tools that facilitate the publishing process and improve the user experience and usability; Codice was built on AJAX (Asynchronous JavaScript + XML). Ajax incorporates standards-based presentation (XHTML and CSS), dynamic display and interaction (Document Object Model), data interchange and manipulation (XML and XSLT), asynchronous data retrieval (XMLHttpRequest) and JavaScript binding everything together [9]. The Ajax engine, allows the user's interaction with Codice to happen asynchronously.

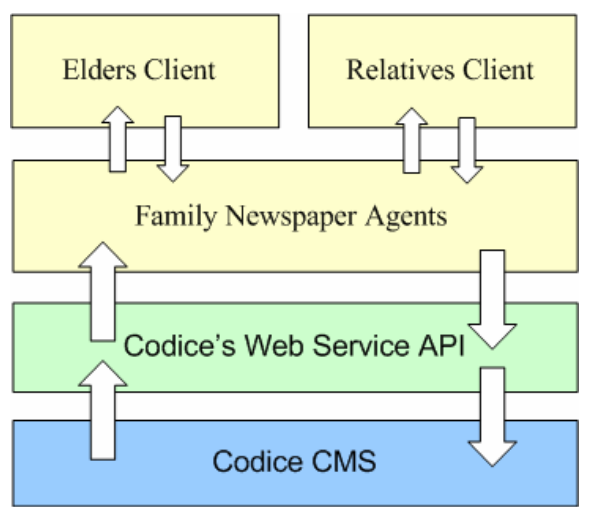

Figure 3. The Family Newspaper architecture.

Codice Web services API. To implement our system we are using the Service-Oriented Computing (SOC) paradigm that utilizes services as fundamental elements for developing applications [10]. A service is an application that exposes its functionality through an application programming interface (API). A Web resource is any type of named information object that is accessible through the Web. Therefore a Web service possesses the characteristics of both a Web resource and a service. It is an application that exposes its functionality through an API, and it is a Web resource that is designed to be consumed by software rather than by a human sitting at a browser [11]. Because services provide an uniform and ubiquitous information distributor for a wide range of computing devices (such a Tablet PCs, PDAs, cellular telephones, or appliances) and software platforms (e.g., LINUX or Windows), we based our architecture on a Web service API layer in order to interact with Codice CMS programmatically.

Elder's Client. This layer is the subsystem that enables elders to create and interact with the electronic family newspaper. The main components of this layer were identified as agents since they have attributes that enabled us to cope with the desirable system feature of facilitating the elder interaction with the system. The metaphor used is that of a personal assistant [12] who seamlessly collaborates with the elder in the same work environment. In this case, autonomous agents proactively help elders to perform difficult tasks, such as using the scanner to publish information on the newspaper, represent the relatives when they are not connected to the system, and to communicate with their relatives. We proposed that autonomous agents not only represent the users living abroad, but may act as proxies to the system's devices (such as Tablet PC, digital cameras or scanners).

Through a Tablet PC, elders can navigate the electronic newspaper and introduce some commands by touching the screen. The system also displays a keyboard so that seniors can easily introduce some text, such as to provide a brief description of a picture. Thus, at the elder's home the Tablet PC can be located in any place where they would like to read the family newspaper. The Tablet PC acts as a server for the system's agents that enable elders to visualize the family newspaper or update it.

Relative's Client. Like the elder's client, the main components of this layer were identified as agents, since they have attributes that enabled us to cope with the desirable system feature of facilitating the interaction with the system. The electronic family newspaper can be accessed by the elder's relative from any computer with a web browser.

Family Newspaper Agents. This layer allows the agents to turn the web services into proactive entities working as peers to serve the elders or their relatives, representing it in the system, composing dynamically the family newspaper. The next section describes the functionality provided by these agents. 


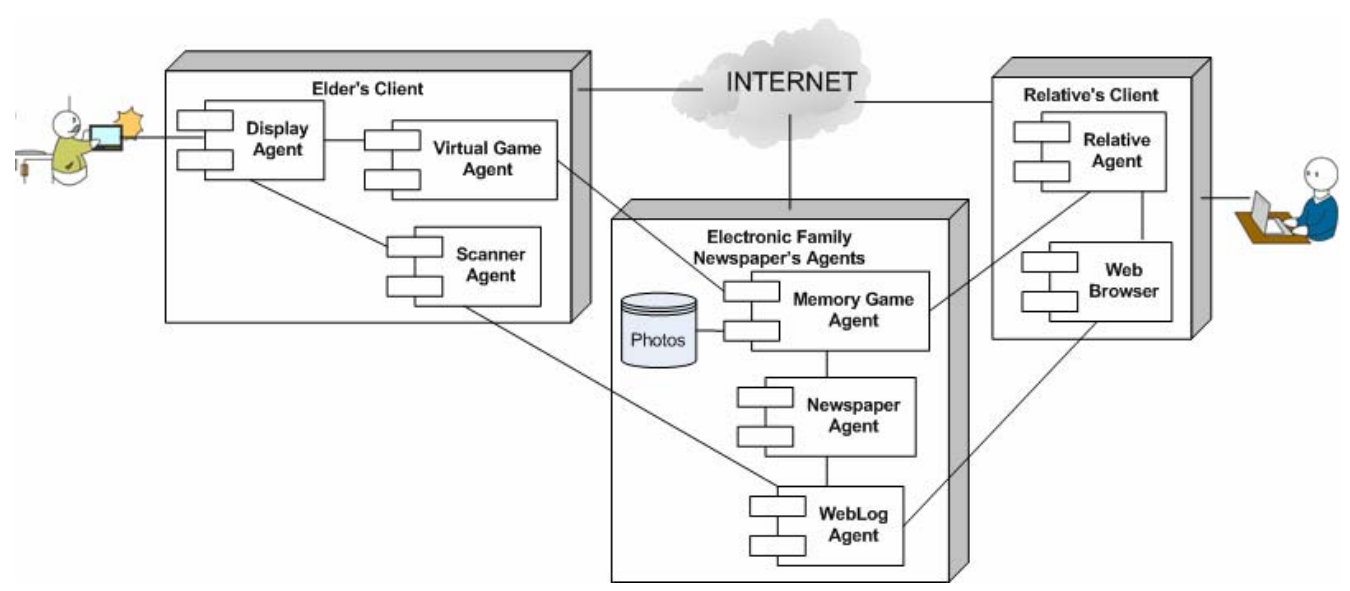

Figure 4. System's components identified as autonomous agents.

\subsection{System Agents}

Envisioning our technological solution as a multiagent systems enabled us to implement a scalable and loosely-coupled system in which by means of autonomous agents we can add new functionality to the system (i.e. new games), integrate new devices (i.e. video cameras) and other people with whom the user may want to be connected, such as close family friends.

The main system's agents (see figure 4) are described next:

The Newspaper Agent: This agent is aware of new entries in the Codice weblog to build or update the newspaper. To monitor and collect the weblog changes, the newspaper agent was built as content a syndication reader powered by the RSS (RDF Site Summary - formerly called Rich Site Summary) standard which is an application of the eXtensible Markup Language (XML) that adheres to the World Wide Web Consortium's Resource Description Framework (RDF) and is a method of describing news or other Web content that is available for syndication or distribution from an online publisher [13], in this case it is accessible through the Codice APIs, which generate XML when a change occurs in the family newspaper.

Weblog agent: It acts as a proxy to the Codice's APIs by enabling users to post information into the Weblog. This agent receives information directly from the elder's relatives or from the agents that help elders to contribute to the family newspaper (see figure 5), such as the Scanner Agent.

Display agent: It is a proxy to the display. It has control of what and when the information is presented in the Tablet PC. For instance, when the display agent is notified that a new entry in the newspaper is available, it automatically opens the family newspaper application.

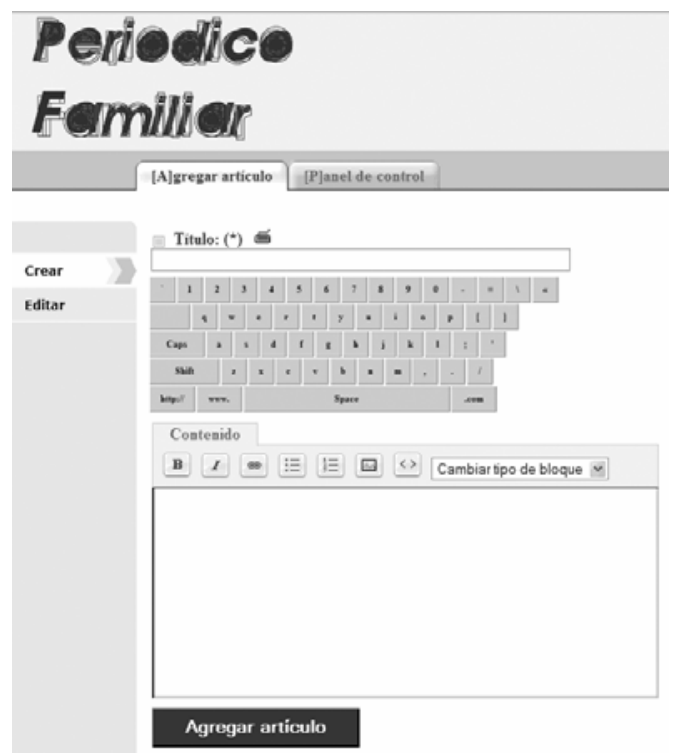

Figure 5. Elder's easy entry text interface.

Scanner Agent: The images and text provided by the elder can be loaded through a scanner. For this, the system provides an agent acting as a proxy to these devices. When the elder scans a document or picture, the Scanner Agent sends the image to the weblog Agent in order to be posted on the weblog and then added to the family newspaper.

Memory Game Agent: When the elder joins the Entertainment section, he or she is presented with several activities, such as the Memory Game. The Memory Game Agent is a server application that monitors the movements of the players, and validates them. It also maintains a database with images and a 
brief story describing them. If the elder chooses to play, this agent will generate a set of cards with the images posted in the weblog, as illustrated in Figure 6.

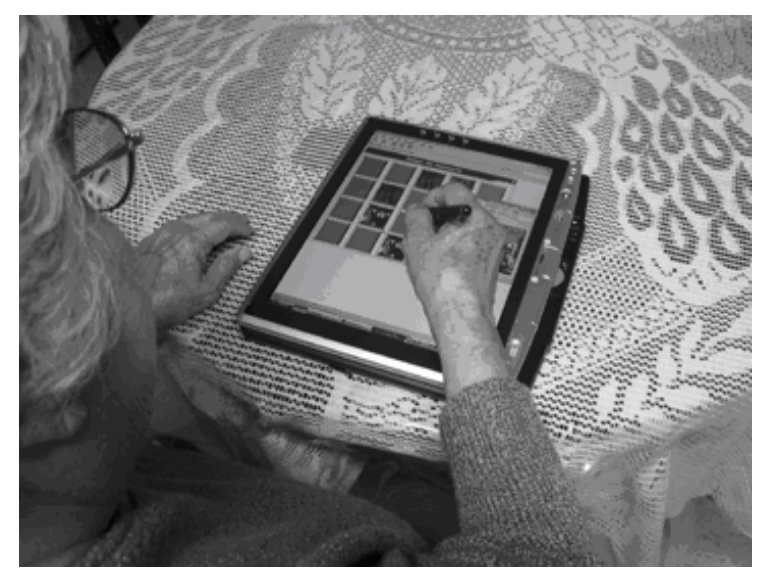

Figure 6. The Memory Game - Entertainment Section.

Virtual Player Agent: This is a companionship agent. If the elder chooses to play the memory game with one of his relatives, the memory game agent will generate a set of cards containing images related with that particular person. Both, the elder and the virtual player agent will make alternate movements. When a pair of cards is matched, the Virtual Player Agent will display a brief story related to that card's image as illustrated in Figure 7. This agent is visually represented by a relative's photograph. When this agent perceives that the person being represented is connected, it invites him to joining to the game. Thus, the relative realizes the elder is thinking about him. If the relative decides to join to the game, the virtual player agent will cede control to him, and the photograph of the relative will be emphasized to indicate the real relative is playing the game.

Real Player Agent: If a relative decides to join a game of memory with the elder, the Real Player Agent is started. Then, it is connected to the game server, which is the Memory Game Agent. The Real Player Agent has an IM client through which the user can maintain contact with the elder while they are playing.

Through a sample application, we describe next how these components interact to support emotional and social ties of elders and their family.

\subsection{Sample application}

We revisit Scenario 1 to illustrate the functionality of the system architecture. Figure 8 illustrates how the system's components interact to support this scenario: While Mario is at his school, he loads in the weblog a review he wrote for his father of the latest soccer game of the Mexican league. The Newspaper Agent is aware that a change was made to the weblog, and updates the family newspaper. Then, it notifies the family Display Agent that the newspaper is available. The Display agent sounds an alarm to advertise that the newspaper has news. Thus, while Mr. Valenzuela is eating his breakfast, he approaches the display and selects the Sports section. As Mr. Valenzuela realizes that his son would like to read some comments from some of the players published in yesterday's newspaper, he scans the note. For this, he touches some buttons on the system to address the scanner system. Then, Mr. Valenzuela chooses to load the note. The order is interpreted by the Weblog Agent that posts the note. Finally, the Newspaper Agent becomes aware of the new change in the weblog, and then, modifies the newspaper.

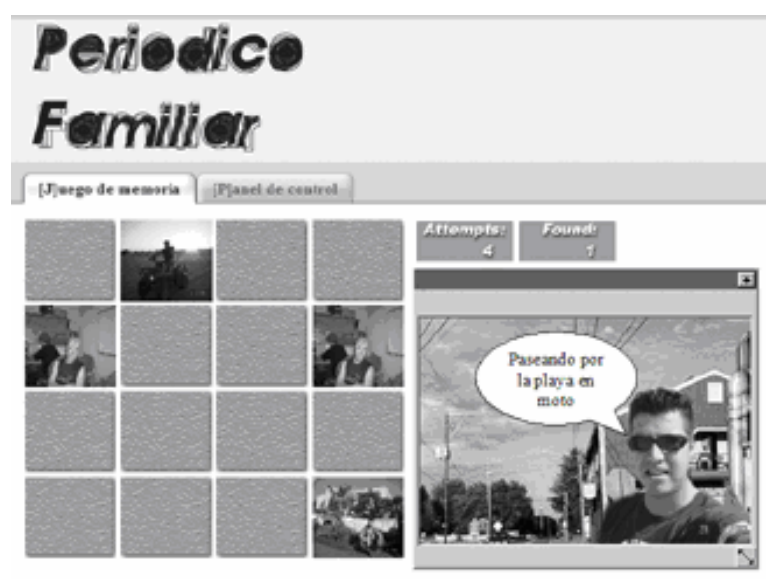

Figure 7. The Virtual Player - Entertainment Section.

\section{Conclusion and future work}

We have presented the design of a system to help elders to incorporate them to the social networks that have become fragmented due to the distance between them and their families living abroad. We centered our design in three main aspects: 1) Provide an easy to use interface for elders. For this, the presentation of the shared information is presented in the format of a newspaper, which is a concept already known by any user. For facilitating the elders' interaction with the family newspaper, we propose they access it through a Tablet-PC with a touch screen, in which there are several autonomous agents as personal assistants. 2) Make the system accessible for the elders' relatives from any computer. The system uses web-based technology, such as weblogs which is a tool already used by many people to share personal information with others. 3) Designing our system in such a way facilitates its extension and evolution. For this, we used autonomous agents as the main design 


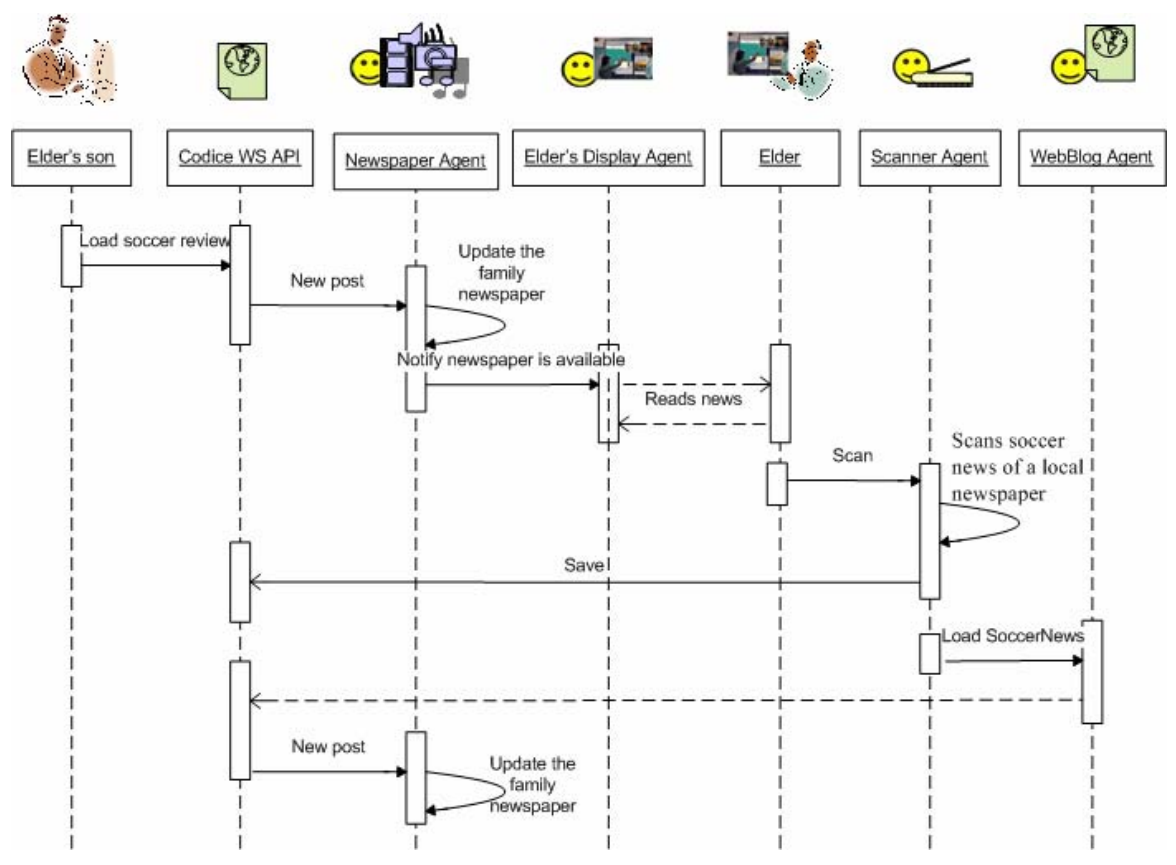

Figure 8. Interaction of the system components.

constructors of the system. In regards to this aspect, we are planning to extend the system's functionality to provide "reminders" to users. For instance, we found from our study that elders' family make phone calls more frequently at the beginning of their separation. However as times goes by, elders and their families might experience more infrequent interactions, their relationships get disrupted and this causes sadness to both parties. To remedy this, agents can be aware of this situation and decide when to send a "reminder" to one or more of the relatives so that the emotional ties remain among them.

\section{References}

1. CONAPO, There are in Mexico 7.9 millions of elders. 2004.

2. Morris, M., et al., New Perspectives on Ubiquitous Computing from Ethnographic Study of Elders with Cognitive Decline. Lecture Notes in Computer Science, 2003. 2864: p. 227-242.

3. Bassuk, S.S., T.A. Glass, and L.F. Berkman, Social Disengagement and Incident Cognitive Decline in Community-Dwelling Elderly Persons. Ann Intern Med, 1999. 131(3): p. 165-173.

4. McCracken, G., The long interview. 1988, Newbury Park, CA, USA: SAGE Publications.

5. Keller, I., W. van der Hoog, and P.J. Stappers, Gust of me: reconnecting mother and son. Pervasive Computing, IEEE, 2004. 3(1): p. 22-27.

6. Fujita, H. and K. Nishimoto. Lovelet: a heartwarming communication tool for intimate people by constantly conveying situation data. in CHI '04 extended abstracts on Human factors in computing systems. 2004. Vienna, Austria: ACM Press.

7. Furlong, M.S., An electronic community for older adults: The seniornet network. Journal of Communication, 1989. 39(3): p. 145-153.

8. Carroll, J.M., Making Use: Scenario-Based Design of Human-Computer Interactions. 2000: MIT Press. 376.

9. Garrett, J.J., Ajax: A New Approach to Web Applications available at http://www.adaptivepath.com/. 2005.

10.Papazoglou, M.P., Service -Oriented Computing: Concepts, Characteristics and Directions, in Proceedings of the Fourth International Conference on Web Information Systems Engineering. 2003, IEEE Computer Society. p. 3.

11. Manes, A.T., Web Services Basics, in Web Services: A Manager's Guide. 2003, Addison-Wesley Professional. p. 27-45.

12. Maes, P., Agents that reduce work and information overload Commun. ACM 199437 (7 ): p. 30-40

13. Çelikbas, Z., What is RSS and how can it serve libraries?, in Info To Go Navigating the Internet. 2004. 